\title{
Intrahepatic Portal Vein Aneurysm with Concurrent Hepatocellular Carcinoma
}

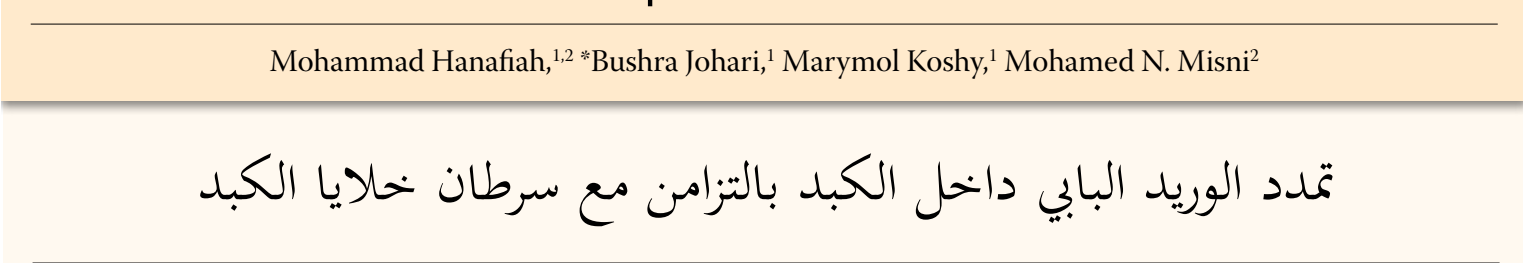

$$
\text { محمد حنافية، بشرى جوهاري، ميريمول كوشي، محمد مسني }
$$

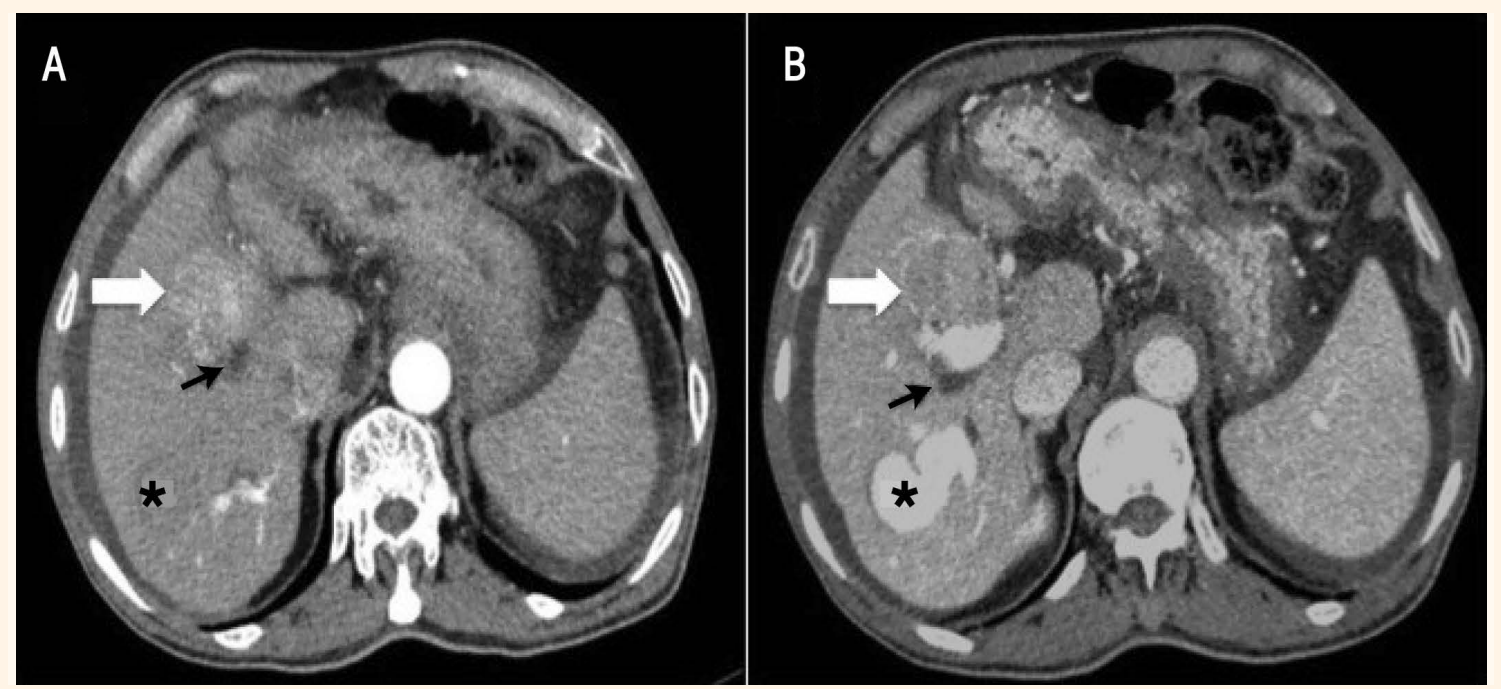

Figure 1A \& B: Selected axial computed tomography images showing a concurrent portal venous aneurysm (asterisks) and a mass in segment $\mathrm{V}$ of the liver, most likely a hepatocellular carcinoma, in a 70-year-old male. The mass (white arrows) demonstrated (A) typical heterogeneous enhancement in the arterial phase and (B) washout in the portal venous phase. Additionally, it caused dilatation of the proximal intrahepatic biliary ducts (black arrows).

A 7O-YEAR-OLD MALE WITH A NINE-YEAR history of chronic hepatitis $B$ infection and liver cirrhosis was admitted to the Kuala Lumpur General Hospital in Kuala Lumpur, Malaysia, in November 2012 with chills and jaundice. A physical examination revealed a distended abdomen without tenderness or masses. There were no other signs of chronic liver disease and the patient was haemodynamically stable. Liver function tests revealed elevated total bilirubin $(172.0 \mu \mathrm{mol} / \mathrm{L})$, alkaline phosphatase (163.0 U/L) and gamma-glutamyl transferase (134.0 U/L) levels. Alanine aminotransferase, white cell count and C-reactive protein levels were within normal limits. However, the patient's serum $\alpha$-fetoprotein levels were elevated $(919.3 \mathrm{ng} / \mathrm{mL})$.

An ultrasound examination of the abdomen showed a cirrhotic liver with fusiform dilatation of the right portal vein. Contrast-enhanced abdominal computed tomography $(\mathrm{CT})$ confirmed the presence of a fusiform aneurysm of the distal right portal vein [Figure 1]. The aneurysm measured $2.2 \mathrm{~cm}$ in diameter and the remainder of the right portal vein was also diffusely dilated. The main portal vein and its left branch, as well as the hepatic veins, were not dilated. These veins were patent with no filling defects to suggest thrombosis. The spleen was mildly enlarged $(14.5 \mathrm{~cm})$ and there were multiple varices in the perigastric region and splenic hilum. An illdefined mass in segment $\mathrm{V}$ of the liver was observed which demonstrated heterogeneous enhancement in the arterial phase and relative contrast washout in the portal venous phase. The greatest diameter of the enhancing component of the lesion in the arterial phase was $4.0 \mathrm{~cm}$. There was extrinsic compression of the mass on the adjacent right intrahepatic duct resulting in dilatation of the proximal biliary system. A 


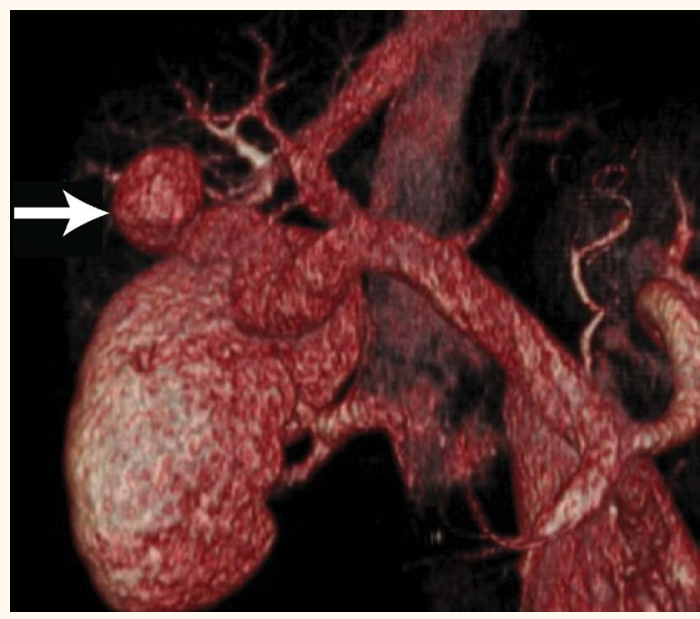

Figure 2: Complementary three-dimensional computed tomography volume-rendered image showing fusiform aneurysmal dilatation at the distal part of the right portal vein (arrow) in a 70-year-old male with concurrent hepatocellular carcinoma. The remainder of the right portal vein was also diffusely dilated.

complementary three-dimensional (3D) CT volumerendered image was constructed to provide a clearer view of the aneurysm [Figure 2].

Given the patient's history of chronic hepatitis B infection, liver cirrhosis and elevated $\alpha$-fetoprotein levels, as well as the enhancement pattern of the mass, a diagnosis of hepatocellular carcinoma (HCC) was made. The patient unfortunately deteriorated clinically while in hospital and died due to complications arising from hospital-acquired pneumonia, cholangitis and hepatic encephalopathy.

\section{Comment}

Portal vein aneurysms (PVAs) are extremely rare, with a worldwide prevalence of $0.43 \%$; however, the condition is increasingly detected with modern imaging technology. ${ }^{1}$ In most cases, patients are asymptomatic and the PVA is discovered incidentally during routine imaging. ${ }^{1}$ The aneurysm is located most frequently in the main portal vein and the confluence of the splenic and superior mesenteric veins. ${ }^{2}$ Generally, PVA is divided into congenital and acquired forms; however, the exact aetiology is unclear and remains controversial. The most common cause of acquired PVA is portal hypertension related to chronic liver disease, such as cirrhosis. ${ }^{3-5}$ Long-standing portal hypertension causes intimal thickening with compensatory medial hypertrophy of the portal vein. ${ }^{3}$ With time, the medial hypertrophy is replaced with fibrous tissue leading to a weakening of the vein wall, thus making it susceptible to aneurysmal dilatation. ${ }^{3}$
However, the incidence of portal hypertension and PVA is disproportionate, suggesting the existence of other contributory factors. Other secondary causes of PVA include pancreatitis, trauma and invasive malignancy. ${ }^{1,5}$ To the best of the authors' knowledge, no data yet exist in the available English scientific literature evidencing a direct relationship between HCC and PVA. As such, this remains an exciting avenue to be explored. It is likely these two conditions have an indirect relationship as both are related to chronic liver disease and cirrhosis.

The present case demonstrates the imaging appearances of PVA on CT scans. Doppler ultrasonography and contrast-enhanced abdominal CT scans are reliable methods of diagnosing PVA; furthermore, these imaging techniques can help identify complications and are useful during the follow-up period. ${ }^{1}$ Contrast-enhanced CT allows multi-planar 3D image reconstruction and can clearly demonstrate the size, location and extent of an aneurysm. ${ }^{5}$ Within the PVA, CT or magnetic resonance imaging differentiate slow-flowing blood from thromboses. ${ }^{1}$ More invasive imaging techniques such as direct or indirect portography are other potential confirmatory investigations, particularly if a portocaval fistula is suspected. However, these procedures are not usually necessary. ${ }^{1}$

In general, PVAs require no treatment and careful follow-up is adequate. ${ }^{2,5}$ The decision to treat a PVA depends on its size and location, the existence of symptoms and the presence of thrombosis. ${ }^{2}$ It is important to be familiar with the imaging appearance and nature of PVAs to allow for appropriate management of the condition.

\section{References}

1. Schwope RB, Margolis DJ, Raman SS, Kadell BM. Portal vein aneurysms: A case series with literature review. J Radiol Case Rep 2010; 4:28-38. doi: 10.3941/jrcr.v4i6.431.

2. Iimuro $Y$, Suzumura K, Ohashi K, Tanaka H, Iijima H, Nishiguchi S, et al. Hemodynamic analysis and treatment of an enlarging extrahepatic portal aneurysm: Report of a case. Surg Today 2015; 45:383-9. doi: 10.1007/s00595-014-0882-8.

3. Rafiq SA, Sitrin MD. Portal vein aneurysm: Case report and review of the literature. Gastroenterol Hepatol (N Y) 2007; 3:296-8.

4. Labgaa I, Lachenal Y, Allemann P, Demartines N, Schäfer M. Giant extra-hepatic thrombosed portal vein aneurysm: A case report and review of the literature. World J Emerg Surg 2014; 9:35. doi: 10.1186/1749-7922-9-35.

5. Laurenzi A, Ettorre GM, Lionetti R, Meniconi RL, Colasanti M, Vennarecci G. Portal vein aneurysm: What to know. Dig Liver Dis 2015; 47:918-23. doi: 10.1016/j.dld.2015.06.003. 